\title{
Azithromycin reduces the viability of human bronchial smooth muscle cells
}

\author{
Rodopi Stamatiou ${ }^{1}$, Konstantinos Boukas ${ }^{2}$, Efrosyni Paraskeva ${ }^{1}$, Paschalis-Adam Molyvdas ${ }^{1}$ \\ and Apostolia Hatziefthimiou ${ }^{1}$
}

The macrolide antibiotic azithromycin has an antiproliferative and autophagic effect on rabbit tracheal smooth muscle cells (SMCs). The purpose of this study is to investigate the effect of azithromycin on human bronchial SMCs. Human bronchial SMCs were treated with azithromycin $\left(10^{-5} \mathrm{M}\right)$ in the presence or absence of $10 \%$ fetal bovine serum (FBS). Cell number was estimated using the Cell Titer $96 \mathbf{A Q}_{\text {ueous }}$ One Solution Assay. Induction of autophagy was studied by observation of cell morphology in cells treated or not with the autophagy inhibitor, 3-methyladenine (3-MA), as well as by Lysotracker Red staining of lysosomes. Activation of apoptosis was assessed with flow cytometry after annexin staining. Incubation with azithromycin for 24,48 or $72 \mathrm{~h}$ reduced viability in FBS-deprived cells, as well as cells cultured in FBS-containing medium. Azithromycin treatment resulted in the formation of cytoplasmic vacuoles that could not be prevented by 3-MA. Furthermore, 3-MA did not reverse the effect of azithromycin on the viability of SMCs. There was an increase in the number of lysosomes in cells treated with azithromycin. Finally, azithromycin increased the percentage of early apoptotic cells. In conclusion, azithromycin reduces the viability of human bronchial SMCs possibly by leading to apoptotic cell death.

The Journal of Antibiotics (2010) 63, 71-75; doi:10.1038/ja.2009.125; published online 15 January 2010

Keywords: apoptosis; autophagy; azithromycin; human bronchial SMCs; viability

\section{INTRODUCTION}

Azithromycin is a widely used and effective antibiotic because of its antibacterial and anti-inflammatory actions. In airway diseases, macrolide therapy seems to be very effective. Specifically, azithromycin is used in low dosages, for a long time, in chronic airway diseases such as chronic bronchitis, diffuse panbronchiolitis and bronchial asthma. ${ }^{1-5}$ Airway wall remodeling, which appears in some of these conditions, includes among others, thickening of the reticular membrane, proliferation of the smooth muscle cells (SMCs) and increase in both the number and size of vessels. 6,7

The improvement in the health status of patients treated with macrolides was attributed to the bacteriostatic and anti-inflammatory effects of such antibiotics. However, data available from previous studies in our laboratory show that azithromycin relaxes precontracted rabbit airway smooth muscle. ${ }^{8}$ Furthermore, we have shown that azithromycin causes a dose-dependent reversible reduction of SMCs proliferation accompanied by autophagy in cells isolated from the trachea of adult rabbits. ${ }^{9}$

The purpose of this study is to examine whether azithromycin has the same effect on human bronchial SMCs.

\section{MATERIALS AND METHODS}

\section{Culture of airway SMCs}

Human bronchial SMCs (cc-2576, Lonza Group, Basel, Switzerland) were used. Cells were grown in Dulbecco's modified Eagle's medium/Ham/F12 (DMEM/ F12) containing $10 \%$ fetal bovine serum (FBS), $100 \mathrm{U} \mathrm{ml}^{-1}$ penicillin and $100 \mu \mathrm{g} \mathrm{ml}^{-1}$ streptomycin (Gibco BRL, Gaithersburg, MD, USA; Invitrogen Corporation, Carlsbad, CA, USA) at $37^{\circ} \mathrm{C}$ in a humidified incubator under $5 \%$ $\mathrm{CO}_{2}$. All experiments were carried out with cells on passages 3-6.

\section{Treatment of cells}

Cells were cultured in serum-free medium for $72 \mathrm{~h}$ before further treatment to achieve quiescence. Azithromycin $\left(10^{-5} \mathrm{M}\right.$ ) (Sigma-Aldrich Chemie, Steinheim, Germany) and $10 \%$ FBS were added to cells for $24-72 \mathrm{~h}$ as indicated. Control cells remained in serum-free medium. Azithromycin was dissolved in DMEM/ F12 medium.

Induction of autophagy

The autophagy inhibitor 3-methyladenine (10 mM) (3-MA, Sigma-Aldrich Chemie) was added to the medium $3 \mathrm{~h}$ before the addition of azithromycin. 3-MA was dissolved in water. The cells were observed under a Nikon eclipse TS100 (Nikon, Melville, NY, USA) inverted microscope using a Leica DFC 480 camera (Leica Cameras, Solms, Germany).

${ }^{1}$ Department of Physiology, Medical School, University of Thessaly, Larissa, Greece and ${ }^{2}$ Department of Immunology and Histocompatibility, Medical School, University of Thessaly, Larissa, Greece

Correspondence: Dr A Hatziefthimiou, Department of Physiology, Medical School, University of Thessaly, Biopolis, Larissa 41110, Greece.

E-mail: axatzi@med.uth.gr

Received 16 September 2009; revised 30 November 2009; accepted 1 December 2009; published online 15 January 2010 


\section{Cell number estimation}

Cells were seeded into 96-well plates at a density of 2000 cells per well, allowed to adhere overnight, washed twice with phosphate-buffered saline and incubated in serum-free medium for $72 \mathrm{~h}$ before further treatment. Replicates of three were used for each point. Cell number was assessed using the Cell Titer 96 $\mathrm{AQ}_{\text {ueous }}$ One Solution Assay (Promega, Madison, WI, USA). Briefly, MTS tetrazolium reagent was added directly to each well and incubated for $2 \mathrm{~h}$ at $37^{\circ} \mathrm{C}$. The MTS tetrazolium compound is converted to formazan by living cells. ${ }^{10,11}$ Absorbance was measured at $490 \mathrm{~nm}$ with a reference at $630 \mathrm{~nm}$ in an ELISA plate reader. There was a linear response between cell number and absorbance at $490 \mathrm{~nm}$ (data not shown).

\section{Lysotracker Red staining}

Cells were stained with Lysotracker Red (Molecular Probes, Eugene, OR, USA; Invitrogen Corporation). ${ }^{12}$ Briefly, cells were incubated in $50 \mathrm{~nm}$ Lysotracker Red for $2 \mathrm{~h}$ at $37^{\circ} \mathrm{C}, 5 \% \mathrm{CO}_{2}$, mounted in a drop of phosphate-buffered saline and observed under a Zeiss Axioskop 40 microscope (Carl Zeiss, New York, NY, USA), using a Leica DFC 480 camera (Leica Cameras).

\section{Assessment of apoptosis}

To obtain the proportion of early apoptotic, alive and dead cells, SMCs were stained with Annexin V and 7-AAD using the Beckman Coulter Annexin V-FITC/7-AAD Kit (Beckman Coulter, Fullerton, CA USA). Accordingly, cells were harvested, washed twice with ice-cold $1 \times$ phosphate-buffered saline, cell pellets resuspended in $100 \mu$ ice-cold $1 \times$ binding buffer, and $10 \mu \mathrm{l}$ of Annexin V-FITC solution and $20 \mu \mathrm{l}$ of 7-AAD Viability Dye were added. Cells were mixed gently, incubated for $15 \mathrm{~min}$ in the dark on ice and events were acquired using an FC500 flow cytometer system (Beckman Coulter) equipped with an argon ion laser and analyzed on the FL1 and FL3 channels. Fluorescence thresholds were determined using unstained and single stained cell populations.

\section{Analysis}

In cell viability experiments, each point was carried out in triplicate and the values presented are the mean of independent experiments. All data are expressed as means \pm s.e. and $N$ refers to the number of experiments. Differences between means were analyzed by one-way analysis of variance with statistically significant differences between groups being determined by Bonferroni post hoc test. A comparison is considered significant when the $P$-value is $<0.05$. The statistical analysis was performed using GraphPad Prism 4 (GraphPad Software, San Diego, CA, USA). Image analysis of the images obtained from the Lysotracker Red staining was conducted using Adobe Photoshop 7.0 (www.adobe.com/products/photoshop/family), and results were expressed as intensity values.

\section{RESULTS}

Incubation with $10^{-5} \mathrm{M}$ azithromycin for $24-72 \mathrm{~h}$ of quiescent SMC in serum-free medium significantly reduced the human bronchial SMC number. For example, the number of SMCs in the presence of azithromycin was $59.3 \pm 12.5 \%$ at $24 \mathrm{~h}, 51.7 \pm 6.5 \%$ at $48 \mathrm{~h}$ and $56.2 \pm 6.2 \%$ of control at $72 \mathrm{~h} \quad(P<0.01$ compared with control, Figure 1).

The number of human bronchial SMCs treated with $10^{-5} \mathrm{M}$ azithromycin in the presence of $10 \%$ FBS for 24,48 and $72 \mathrm{~h}$ was also suppressed to $54.2 \pm 6.31,46.4 \pm 1.9$ and $49.5 \pm 4.0 \%$ of control, respectively (Figure 1). On the other hand, the estimated cell number in $10 \% \mathrm{FBS}$ was increased to $118.7 \pm 5.2 \%$ at $24 \mathrm{~h}, 141.2 \pm 9.1 \%$ at $48 \mathrm{~h}$ and $157.4 \pm 9.4$ at $72 \mathrm{~h}$ compared with control (Figure 1).

Treatment of human bronchial SMCs, with $10^{-6}-6 \times 10^{-6} \mathrm{M}$ azithromycin in $10 \%$ FBS-containing or in serum-free medium, did not significantly affect their number (data not shown).

Moreover, treatment of human bronchial SMCs with $10^{-5} \mathrm{M}$ azithromycin for $24 \mathrm{~h}$ both in the presence or absence of FBS resulted to the formation of cytoplasmic vacuoles, reminiscent of autophagy (Figure 2).
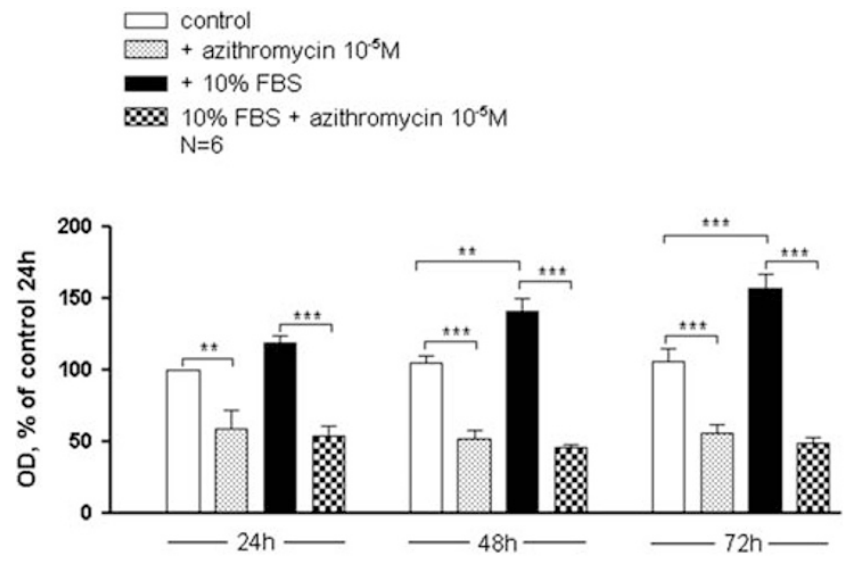

Figure 1 The effect of $10^{-5} \mathrm{M}$ azithromycin on the viability of human bronchial SMCs incubated in 10\% FBS-containing or serum-free medium. Data are presented as mean \pm s.e. $N=6$ is the number of independent experiments. ${ }^{* *} P<0.01$ and ${ }^{* * *} P<0.001$ compared with control or $10 \%$ FBS

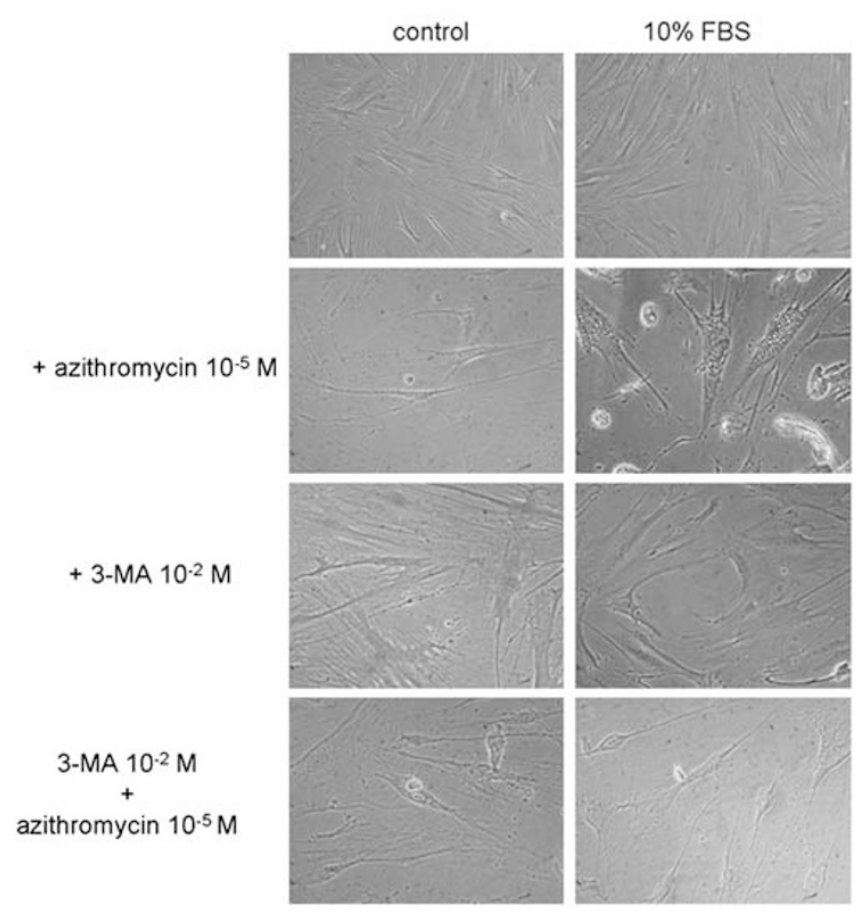

Figure 2 The effect of azithromycin $\left(10^{-5} \mathrm{M}\right)$ on the induction of autophagy in human bronchial SMCs cultured in serum-free or $10 \%$ FBS-containing medium. Where indicated, 3-MA $(10 \mathrm{~mm})$ was added to the cell culture medium.

We assessed whether 3-MA (10 mM), an inhibitor of class III phosphotidylinositol-3-kinases that blocks autophagy, ${ }^{13,14}$ inhibits the effect of azithromycin in these cells. Pretreatment of human bronchial SMCs with 3-MA had no significant effect on the formation of vacuoles (Figure 2). In addition, 3-MA pretreatment did not affect the reduction of SMC numbers induced by azithromycin (Figure 3).

To study the nature of the vacuoles formed in azithromycin-treated cells, we used Lysotracker Red, a fluorescent acidotropic probe for labeling and tracking of lysosomes in live cells. The treatment of SMCs with $10^{-5} \mathrm{M}$ azithromycin for $24 \mathrm{~h}$ increased the luminosity intensity level (Figure 4), indicating an increase in the number of lysosomes. 
Finally, the proportion of early apoptotic, alive and dead cells was studied using Annexin V and 7-AAD staining. Azithromycin increased the percentage of the early apoptotic population from 14.5 to $27.5 \%$ when added to cells in the FBS-free medium and from 6.8 to $20.1 \%$ in cells cultured in FBS-containing medium (Figure 5).
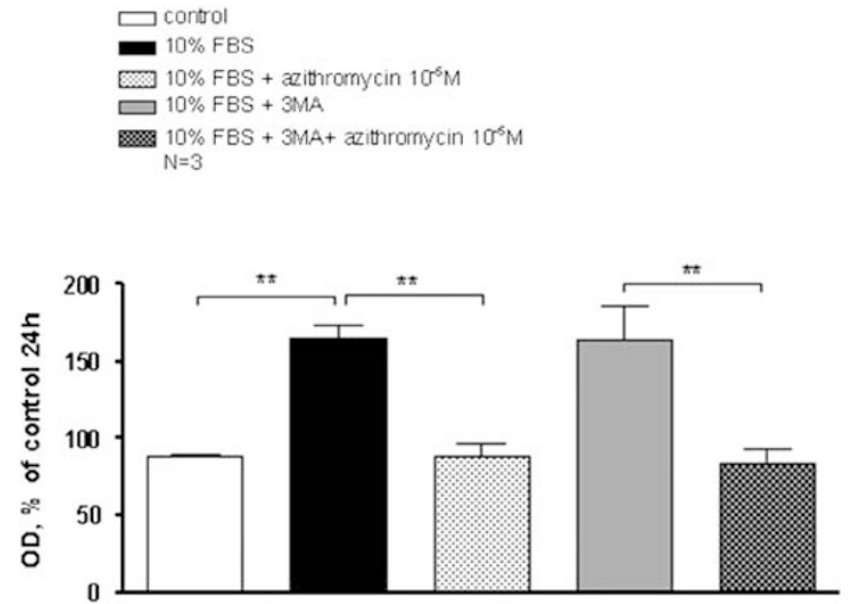

Figure 3 3-MA $(10 \mathrm{~mm})$ does not reverse the effect of azithromycin $\left(10^{-5} \mathrm{M}\right)$ on human bronchial SMC numbers estimated with Cell Titer 96 AQueous One Solution Assay. Data are presented as mean \pm s.e. $N=3$ is the number of independent experiments. ${ }^{* *} P<0.01$ compared with control or $10 \% \mathrm{FBS}$.

\section{DISCUSSION}

The results from this study indicate that azithromycin reduces the viability of human bronchial airway SMCs. This effect is probably mediated by the induction of autophagy that drives the cells to

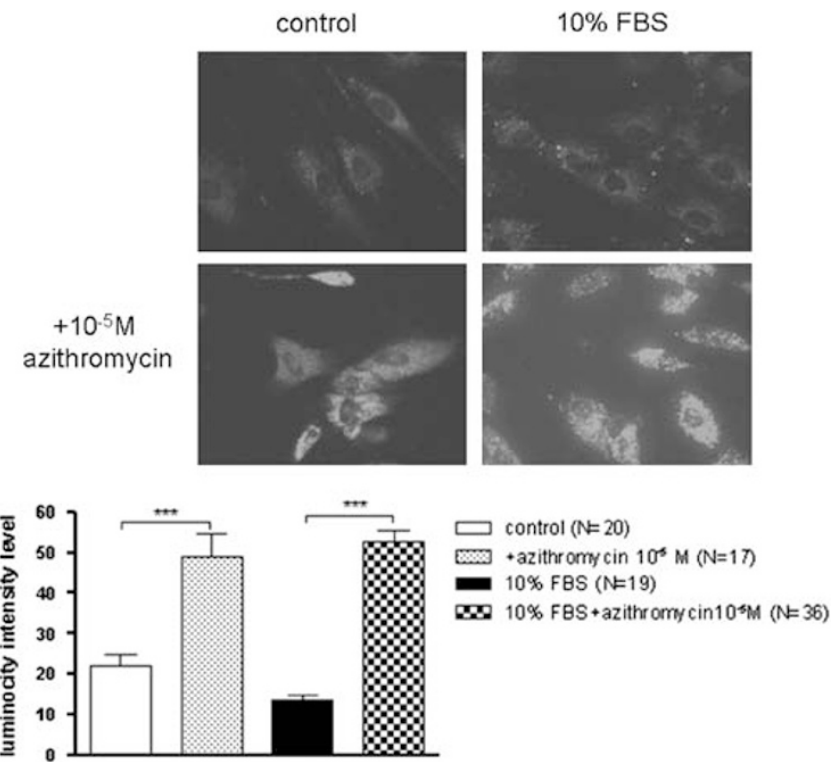

Figure 4 Lysotracker Red staining observed by immunofluorescence microscopy reveals an increase luminosity intensity level in azithromycin $\left(10^{-5} \mathrm{M}\right)$-treated cells. Data are presented as mean \pm s.e. and $N$ is the number of cells. ${ }^{* * *} P<0.001$ compared with control or $10 \%$ FBS.
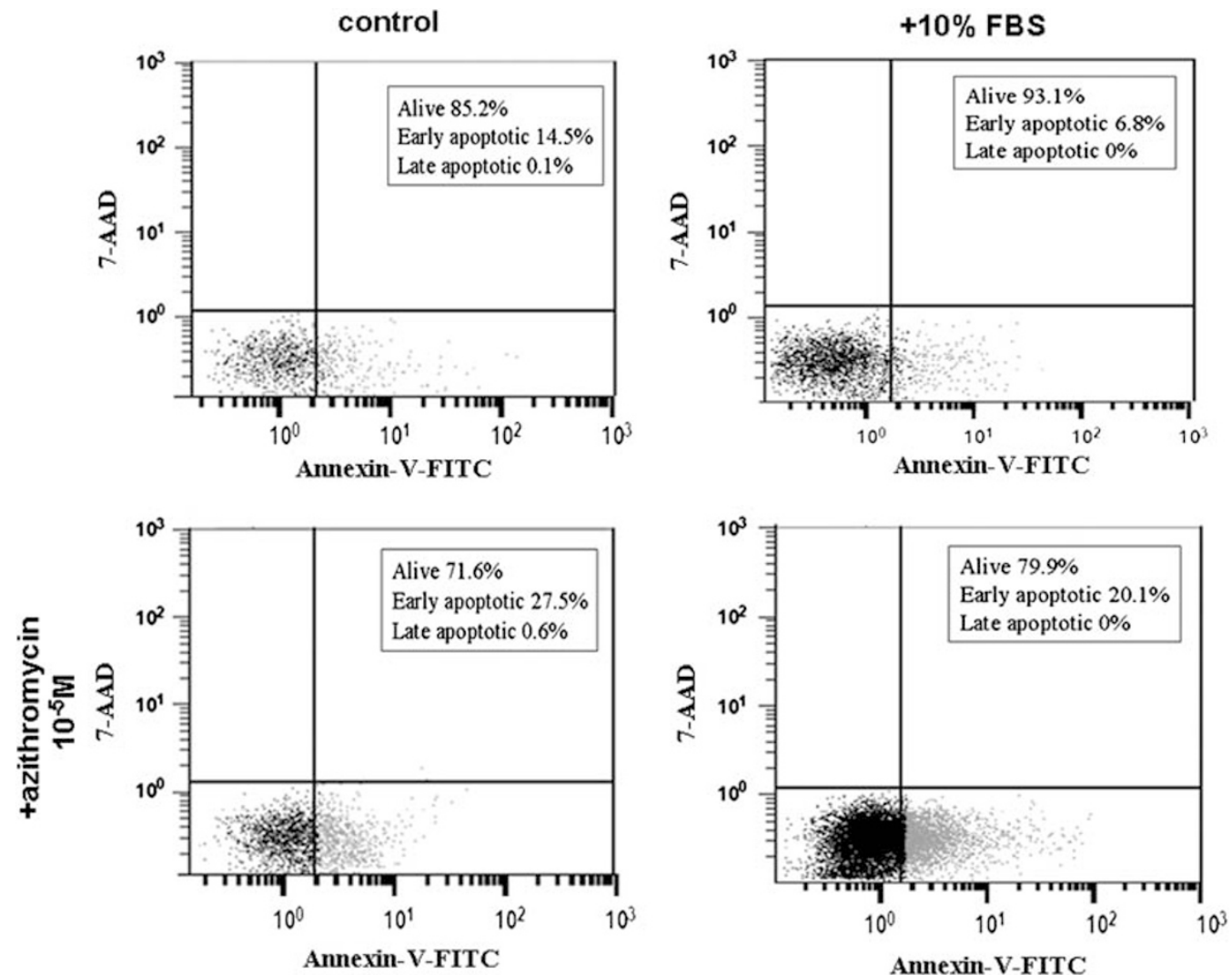

Figure 5 The effect of azithromycin $\left(10^{-5} \mathrm{M}\right)$ in the proportion of early apoptotic, alive and dead SMCs, after staining with Annexin V and 7-AAD, using the Beckman Coulter Annexin V-FITC/7-AAD Kit and analyzed with a FC500 flow cytometer. Each experiment was repeated at least twice. 
apoptosis. Azithromycin decreases the viability of human bronchial SMCs cultured both in medium containing $10 \%$ FBS, as well as in serum-free medium. These results suggest that the effect of azithromycin on these cells is independent of the proliferative status of the cells. Moreover, it does not depend on the duration of treatment with azithromycin, as the effect reaches its maximum at the first $24 \mathrm{~h}$ of incubation in both FBS-fed and serum-starved cells.

There are studies showing that in chronic airway diseases SMC represent a mixed population of two different phenotypes, a contractile (elongated cells) and a synthetic one. These two phenotypes seem to have different responses to stimuli that affect cell proliferation. ${ }^{15-17}$ The percentage of the contractile phenotype in canine tracheal SMCs is about $8.9 \%$, and after 7 days of serum starvation it appears elevated to the point of $18 \%$ of total population. ${ }^{17}$ Therefore, only a minor percentage of the culture population seems to be different. In our experiments, we used cells that were incubated in serum-free medium for $72 \mathrm{~h}$ to synchronize the cells in a nonproliferative status. The population we used seems to be homogeneous. The lack of timedependent effect could be attributed to the fact that azithromycin is a stimulus too intense for these cells, which leads them rapidly to apoptosis after $24 \mathrm{~h}$ of treatment.

There are a few studies on epithelial or SMCs that suggest that macrolide antibiotics may affect cell proliferation. Specifically, roxithromycin, SAR943, a rapamycin analog, as well as rapamycin itself inhibit the proliferation of human coronary artery SMCs, ${ }^{18}$ epithelial and airway SMCs, ${ }^{19}$ and rapamycin also inhibits the proliferation of hepatocytes. ${ }^{20}$ Other macrolides such as erythromycin have been shown to inhibit hypertrophic and metaplastic changes of goblet cells in rat nasal epithelium, as well as inhibit the proliferation of human mononuclear cells. ${ }^{21,22}$ Azithromycin along with clarithromycin are reported to induce apoptosis of activated lymphocytes. ${ }^{23} \mathrm{In}$ previous studies in our laboratory, azithromycin was also observed to cause reversible autophagy in rabbit tracheal SMCs, but not apoptosis, as well as reversible inhibition of the proliferation. ${ }^{9}$ Interestingly, azithromycin seems to affect cell proliferation by inducing both autophagy and apoptosis, with the threshold between the two procedures being affected by cell type.

Azithromycin treatment caused cytoplasmic vacuoles formation. These vacuoles are stained with Lysotracker Red, pointing out the presence of lysosomes and seem to be autophagic. However, the inhibitor of autophagy, 3-MA, did not prevent neither vacuole formation nor the reduction of cell number, caused by azithromycin. Moreover, the percentage of early apoptotic cells increased after azithromycin treatment. These results suggest that azithromycin may induce an intense or fast effect on human bronchial SMCs that leads the cells to extended autophagy and induces apoptosis within the first $24 \mathrm{~h}$ of treatment. Previous studies in our laboratory showed that azithromycin caused a reversible inhibition of rabbit tracheal SMCs proliferation, accompanied by autophagy, but did not induce apoptosis. ${ }^{9}$ However, there is evidence that autophagy and apoptosis are not mutually exclusive. ${ }^{24-26}$ Whether stress conditions will lead the cell to autophagy or apoptosis depends on the nature, intensity and duration of the effect, as well as the cell type and energy condition of the cell at the time that it received the effect. ${ }^{27}$

Furthermore, although azithromycin displayed a dose-response effect on rabbit tracheal SMC proliferation, in concentrations $10^{-6}$ $10^{-5} \mathrm{M},{ }^{9}$ such a dose-dependent effect could not be observed on human bronchial SMCs. In contrast, azithromycin seems to have a none or all effect in human bronchial SMCs, with the concentration $10^{-5} \mathrm{M}$ being the threshold. Interestingly, when azithromycin has been used in clinical studies, the administrated concentration resulted in a lung concentration of $8.93 \mathrm{mgl}^{-1}$, corresponding to $\sim 10^{-5} \mathrm{M}$ of azithromycin. $^{28,29}$ The same concentrations were used when the anti-inflammatory and antibacterial effect of azithromycin was studied. ${ }^{30,31}$

In summary, our results show that treatment of human SMCs with azithromycin reduces cell viability possibly through apoptosis. In addition to the known antibacterial and anti-inflammatory role of azithromycin, this pro-apoptotic effect on human bronchial SMCs might be of clinical significance.

1 Kudoh, S., Azuma, A., Yamamoto, M., Izumi, T. \& Ando, M. Improvement of survival in patients with diffuse panbronchiolitis treated with low-dose erythromycin. Am. J. Respir. Crit. Care Med. 157, 1829-1832 (1998).

2 Oishi, K. et al. Role of interleukin-8 (IL-8) and an inhibitory effect of erythromycin on IL-8 release in the airway of patients with chronic airway diseases. Infect. Immun. 62, 4145-4152 (1994).

3 Shirai, T., Sato, A. \& Chida, K. Effect of 14-membered ring macrolide therapy on chronic respiratory tract infections and polymorphonuclear leukocyte activity. Intern. Med. 34, 469-474 (1995).

4 Miyatake, H. et al. Erythromycin reduces the severity of bronchial hyperresponsiveness in asthma. Chest 99, 670-673 (1991).

5 Cazzola, M., Salzillo, A. \& Diamare, F. Potential role of macrolides in the treatment of asthma. Monaldi. Arch. Chest Dis. 55, 231-236 (2000).

6 Vignola, A. et al. Airway remodelling in asthma. Chest 123, 417S-422S (2003).

7 Jeffery, P. K. Remodeling in asthma and chronic obstructive lung disease. Am. J. Respir. Crit. Care Med. 164, S28-S38 (2001).

8 Daenas, C., Hatziefthimiou, A. A., Gourgoulianis, K. I. \& Molyvdas, P. A. Azithromycin has a direct relaxant effect on precontracted airway smooth muscle. Eur. J. Pharmacol. 553, 280-287 (2006).

9 Stamatiou, R. et al. Azithromycin has an antiproliferative and autophagic effect on airway smooth muscle cells. Eur. Respir. J. 34, 1-11 (2009).

10 Cory, A. H., Owen, T. C., Barltrop, J. A. \& Cory, J. G. Use of an aqueous soluble tetrazolium/formazan assay for cell growth assays in culture. Cancer Commun. 3, 207212 (1991).

11 Berridge, M. V. \& Tan, A. S. Characterization of the cellular reduction of 3-(4, 5-dimethylthiazol-2-yl)-2,5-diphenyltetrazolium bromide (MTT): subcellular localization, substrate dependence, and involvement of mitochondrial electron transport in MTT reduction. Arch. Biochem. Biophys. 303, 474-482 (1993).

12 Shen, Z. Y. et al. Autophagy and endocytosis in the amnion. J. Struct. Biol. 162, 197-204 (2008)

13 Seglen, P. O. \& Gordon, P. B. 3-Methyladenine: specific inhibitor of autophagic/ lysosomal protein degradation in isolated rat hepatocytes. Proc. Natl Acad. Sci. USA 79, 1889-1892 (1982).

14 Petiot, A., Ogier-Denis, E., Blommaart, E. F., Meijer, A. J. \& Codogno, P. Distinct classes of phosphatidylinositol 3 '-kinases are involved in signalling pathways that control macroautophagy in HT-29 cells. J. Biol. Chem. 275, 992-998 (2000).

15 Hirst, S. J., Walker, T. R. \& Chilvers, E. R. Phenotypic diversity and molecular mechanisms of airway smooth muscle proliferation in asthma. Eur. Respir. J. 16, 159-177 (2000)

16 Bentley, J. K. \& Hershenson, M. B. Airway smooth muscle growth in asthma: proliferation, hypertrophy, and migration. Proc. Am. Thorac. Soc. 5, 89-96 (2008).

17 Halayko, A. J. \& Amrani, Y. Mechanisms of inflammation-mediated airway smooth muscle plasticity and airways remodeling in asthma. Physiol. Neurobiol. 137, 209-222 (2003).

18 Tomita, H. et al. Roxithromycin is an inhibitor of human coronary artery smooth muscle cells proliferation: a potential ability to prevent coronary heart disease. Atherosclerosis 182, 87-95 (2005).

19 Fujitani, Y. \& Trifilieff, A. In vivo and in vitro effects of SAR 943, a rapamycin analogue, on airway inflammation and remodeling. Am. J. Respir. Crit. Care Med. 167, 193-198 (2003).

20 Buitrago-Molina, L. E. et al. Rapamycin delays tumor development in murine livers by inhibiting proliferation of hepatocytes with dna damage. Hepatology 50, 2 (2009).

21 Takahashi, Y., Shimizu, T. \& Sakakura, Y. Effects of indomethacin, dexamethasone, and erythromycin on endotoxin-induced intraepithelial mucus production of rat nasal epithelium. Ann. Otol. Rhinol. Laryngol. 106, 683-687 (1997).

22 Roche, Y., Gougerot-Pocidalo, M. A., Fay, M., Forest, N. \& Pocidalo, J. J. Macrolides and immunity: effects of erythromycin and spiramycin on human mononuclear cell proliferation. J. Antimicrob. Chemother. 17, 195-203 (1986).

23 Mizunoe, S. et al. Clarithromycin and azithromycin induce apoptosis of activated lymphocytes via down-regulation of Bcl-xL. Int. Immunopharmacol. 4, 1201-1207 (2004).

24 Cui, Q., Tashiro, S., Onodera, S., Minami, M. \& Ikejima, T. Autophagy preceded apoptosis in oridonin-treated human breast cancer MCF-7 cells. Biol. Pharm. Bull. 30, 859-864 (2007)

25 Yan, C. H. et al. Autophagy is involved in cytotoxic effects of crotoxin in human breast cancer cell line MCF-7 cells. Acta. Pharmacol. Sin. 28, 540-548 (2007). 
26 Shacka, J. et al. Bafilomycin A1 inhibits cloroquine-induced death of celebellar granule neurons. Mol. Pharmacol. 69, 1125-1136 (2006).

27 Canu, N. et al. Role of the autophagic-lysosomal system on low potassiuminduced apoptosis in cultured cerebellar granule cells. J. Neurochem. 92, 12281242 (2005).

28 Shimizu, T., Shimizu, S., Hattori, R., Gabazza, E. C. \& Majima, Y. In vivo and in vitro effects of macrolide antibiotics on mucus secretion in airway epithelial cells. Am. J. Respir. Crit. Care Med. 168, 581-587 (2003).
29 Danesi, R. et al. Comparative distribution of azithromycin in lung tissue of patients given oral daily doses of 500 and $1000 \mathrm{mg}$. J. Antimicrob. Chemother. 51, 939-945 (2003).

30 Patel, K. B. et al. Comparison of bronchopulmonary pharmacokinetics of clarithromycin and azithromycin. Antimicrob. Agents Chemother. 40, 2375-2379 (1996).

31 Phaff, S. J., Tiddens, H. A., Verbrugh, H. A. \& Ott, A. Macrolide resistance of Staphylococcus aureus and Haemophilus species associated with long-term azithromycin use in cystic fibrosis. J. Antimicrob. Chemother. 57, 741-746 (2006). 